\title{
Bewertung von Abfallströmen aus Gebäudeabbrüchen in Wien auf Grundlage von Bildmatching-basierter Veränderungsdetektion
}

\author{
Fritz Kleemann · Hubert Lehner · Anna Szczypińska · Jakob Lederer · Johann Fellner
}

Online publiziert: 22. Januar 2018

(C) Der/die Autor(en) 2018. Dieser Artikel ist eine Open-Access-Publikation.

\begin{abstract}
Zusammenfassung In urbanen Gebieten resultieren große Abfallströme aus Gebäudeabbrüchen. Obgleich gesamthafte Zahlen über den nationalen Anfall an Baurestmassen verfügbar sind, fehlen auf regionaler Ebene oft Daten zu Abbruchabfällen oder sind lückenhaft. Eine alternative Möglichkeit, die Menge und Zusammensetzung von Abbruchabfällen indirekt zu berechnen, besteht darin, abgebrochene Gebäudevolumina mit spezifischen Materialintensitäten $\mathrm{zu}$ multiplizieren. Da Informationen zur Abbruchaktivität (Anzahl der abgebrochenen Gebäude bzw. abgebrochenes Gebäudevolumen) auf Basis von statistischen Daten bzw. behördlichen Aufzeichnungen oft unvollständig sind, wird in der vorliegenden Studie eine alternative Methode präsentiert, die es mithilfe von Bildmatching-ba-

$\mathrm{m}^{3} /$ Jahr basierend auf Aufzeichnungen über Abbruchanzeigen bzw. 2,8 Mio. $\mathrm{m}^{3} / \mathrm{Jahr}$ unter Anwendung Bildmatching-basierter Veränderungsdetektion). Demzufolge führt die Verwendung der behördlichen Aufzeichnungen zu einer Unterschätzung der Abbruchaktivität und des damit im Zusammenhang stehenden Abfallaufkommens. Der präsentierte Ansatz ermöglicht einerseits die Plausibilisierung existierender Daten und andererseits die Erhebung neuer Daten, wenn diese nicht vorhanden sind. Weiters bietet die Methode die Grundlage für ein kontinuierliches Monitoring des Gebäudebestands und dessen dynamischer Entwicklung. Dies wiederum ermöglicht Prognosen von Materialflüssen im Bausektor, welche zur Förderung regionaler Materialkreisläufe genutzt werden kann.
\end{abstract} sierter Veränderungsdetektion erlaubt, die Abbruchaktivität zu erheben und damit Vergleichsdaten zu Statistiken zu generieren. In weiterer Folge können Aussagen über Menge und Qualität von Abbruchabfällen in Wien getroffen werden. Die angewandte Methode erlaubt es weiters, bei den Behörden nicht angezeigte Abbruchaktivitäten zu identifizieren. Die Ergebnisse hinsichtlich des abgebrochenen Gebäudevolumens in Wien unterscheiden sich je nach Datenquelle signifikant (1,7 Mio.

DI Dr. F. Kleemann $(\bowtie)$.

Mag. DI Dr. J. Lederer •

Ass.-Prof. DI Dr. J. Fellner

Christian Doppler Labor für

Anthropogene Ressourcen,

Technische Universität Wien,

Karlsplatz 13/226, 1040 Wien,

Österreich

fritz.kleemann@tuwien.ac.at

DI H. Lehner · A. Szczypińska, MSc.

(Eng.)

Magistratsabteilung 41,

Stadtvermessung Wien,

Muthgasse 62, 1190 Wien, Österreich

\section{Assessment of waste streams from building demolition in Vienna based on image-matching change detection}

Abstract Major waste streams in urban areas result from the demolition of buildings. In case of lacking data on demolition waste generation at the regional level, the quantity and composition of demolition waste from buildings can be estimated by multiplying the volume of demolished buildings by their specific material intensities. Information about building demolition (number of demolished buildings, demolished building volume) based on statistical data or other official records are often incomplete. Hence, this paper presents an alternative approach for validating demolition statistics (number and volume of demolished buildings) and subsequently demolition waste generation by applying change detection based on image matching using the city of Vienna (Austria) as a case study. Based on this technique, building demolition activities not reported to statistical municipal departments can be identified. Results for 2013/14 show that in the city of Vienna, demolition statistics yield a total volume of $1.7 \mathrm{M} \mathrm{m}^{3} / \mathrm{a}$ demolished building volume, while change detection based on image matching yields a total volume of $2.8 \mathrm{M} \mathrm{m}^{3} / \mathrm{a}$. Consequently, demolition waste generation figures solely based on statistical data would significantly underestimate the total waste generation. The presented approach is a useful tool for validating existing data on demolition waste generation and demolition statistics and can also be used when these data sets are not existent at all. Furthermore, the approach serves as a basis for continuous monitoring of the building stock and its dynamics. The latter is regarded as a prerequisite for the prognosis of material flows and for the promotion of a circular economy in the building sector.

\section{Einleitung}

Zwischen 20 und $30 \%$ des Abfalls in Österreich sind auf Bau- und Abbruchaktivitäten zurückzuführen (Aushubmaterialien ausgenommen) (BMLFUW $2011,2015)$. Auch auf europäischer Ebene zeichnet sich ein ähnliches Bild ab (European Comission 2014). Dadurch besteht ein hohes Potenzial, durch Recycling von Baurestmassen einerseits Primärressourcen und andererseits Deponievolumen einzusparen. Die Recyclingziele der EU sehen vor, dass bis 2020 mindestens $70 \%$ der nichtgefährlichen Baurestmassen (Aushubmaterial ausgenommen) zur Wiederverwendung oder Wiederverwertung vorbereitet oder anderweitig rückgewonnen werden sollen (European Parliament \& Council of the European Union 2008). Um dies zu erzielen und zu berechnen, sind Informationen über Menge und Qualität von Materialien, die ins Abfallregime gelangen, wichtig. Im Un- 
terschied zu vielen anderen Abfallarten (Verpackungsabfälle, Elektroaltgeräte etc.), spielt sich das Abfallmanagement von Bau- und Abbruchabfällen hauptsächlich auf regionaler und weniger auf nationaler oder gar internationaler Ebene ab. Der Grund dafür ist der limitierte Transportradius $(<50 \mathrm{~km})$ der mineralischen Abfallfraktionen, welche massemäßig über $90 \%$ darstellen. Um dieser Regionalität - auch hinsichtlich möglicher Optimierungspotenziale im Abfallmanagement - gerecht zu werden, bedarf es regionaler Daten zu Aufkommen und Qualität der relevanten Abfallfraktionen. Eine weitere Besonderheit der Materialien im Baubereich ist deren lange und sehr unterschiedliche Nutzungsdauer, weshalb es schwierig ist, Annahmen zur Lebensdauer zu treffen und mithilfe dieser Abfallmengen und -zusammensetzungen abzuschätzen.

Bei der Einhaltung von Recyclingzielen spielen Abbruchabfälle aus Hochbauten aus zweierlei Gründen eine besondere Rolle. Einerseits sind Hochbauten in ihrer Materialzusammensetzung deutlich komplexer als die meisten Infrastrukturbauten (Straßen, Schienen, Netze) und damit generell schwieriger zu recyceln. Andererseits ist in Städten der Gebäudebestand im Vergleich zu Infrastrukturen dominant und der Materialverbrauch und der Anfall an Bauund Abbruchabfällen höher als in ländlichen Regionen. Eine Unterscheidung der Abfälle hinsichtlich ihres Ursprungs ist aus den derzeitigen Aufzeichnungen in Österreich nicht möglich und der Anteil von Abfällen aus Gebäudeabbrüchen im Vergleich zu Abfällen aus dem Tiefbau ist schwer abzuschätzen bzw. $\mathrm{zu}$ verifizieren.

Das Aufkommen und der Umgang mit Bau- und Abbruchabfällen wurden in den letzten Jahren sowohl auf nationaler als auch internationaler Ebene intensiv beforscht (Yuan und Shen 2011). Dabei reichen die gesetzten Schwerpunkte von Fallstudien zu einzelnen Gebäuden bis hin zu globalen Materialflussanalysen unterschiedlicher Baumaterialien. Im Folgenden finden sich einige dieser Studien. Die Bandbreite der verwendeten Datengrundlagen ist dabei groß und reicht von der Analyse historischer Daten bis zur Verwendung von BIM (Building Information Modeling) Daten zur Abschätzung von Bauund Abbruchabfällen.

$\mathrm{Zu}$ den Arbeiten, die darauf abzielen, Bau- und Abbruchabfälle auf nationaler oder regionaler Ebene zu charakterisie- ren, zählen Hashimoto et al. (2009), wobei Materiallager in Gebäuden und Infrastruktur und daraus resultierende Abfälle für Japan abgeschätzt wurden. Zur Berechnung der Abfallmengen aus dem Gebäudebereich wurden dabei Materialintensitäten je Fläche angenommen. Bergsdal et al. (2007) präsentierten einen Ansatz, welcher auf unterschiedlichen Aktivitätslevels im Bausektor basiert. Je intensiver die Bauaktivität, desto größere Mengen an Bau- und Abbruchabfällen sind zu erwarten. Die Arbeit zielte darauf $\mathrm{ab}$, Mengenabschätzungen zu liefern, um das Abfallwirtschaftssystem hinsichtlich Kapazität und Technik für die Zukunft zu rüsten. Fatta et al. (2003) schätzten Bau- und Abbruchabfälle auf Basis ausgestellter Abbruchlizenzen und anfallender Materialien je $\mathrm{m}^{2}$ Baufläche für Griechenland ab. Cochran und Townsend (2010) wendeten die Methode der Materialflussanalyse für Bau- und Abbruchabfälle für eine Region der USA an, wobei bestimmte Lebensdauern für unterschiedlich eingesetzte Baumaterialien angenommen wurden.

Tanikawa und Hashimoto (2009) entwickelten ein 4-dimensionales GIS (Geoinformationssystem), um räumliche und zeitliche Dynamiken bei der Akkumulation von Materialien in Gebäuden und Infrastruktur zu untersuchen. Dabei standen zwei Beispielregionen in Japan und Großbritannien im Fokus.

Hu et al. (2010) modellierten sowohl das Materiallager in Pekings Wohngebäuden als auch Input- und Outputflüsse basierend auf der Materialzusammensetzung unterschiedlicher Gebäudetypen. De Melo et al. (2011) schätzten Bau- und Abbruchabfälle auf Basis der Bauaktivität und assoziierter Lkw-Fahrten für Lissabon ab. Ziel dabei war es, das Abfallmanagement zu verbessern und illegale Ablagerungen zu vermeiden.

Die Arbeit von Solís-Guzmán et al. (2009) untersuchte Abfallmengen beim Abbruch und bei der Konstruktion von Einzelgebäuden. Ihr Ansatz basiert auf Mengenlisten sowie Abschätzungen zu abgebrochenen Gebäudevolumina sowie Verpackungsmaterialien. Cheng und Ma (2013) verwendeten Building Information Modeling (BIM), um die Materialität von Gebäuden abzuschätzen. Ihr Ansatz ermöglicht es, Informationen über in einzelnen Gebäuden verbaute Materialien und Abfallmengen zu generieren. So sollen Recycling und Wiederverwendung effizient geplant und Entsorgungskosten errechnet werden. Voraussetzung für die Anwendbarkeit dieser Methode ist die vollständige Erfassung der Materialien im BIM.

Während viele der genannten Studien einen starken Fokus auf die Entwicklung von Modellen zur Simulation von Materiallagern und -flüssen setzten, scheinen die dahinterliegenden Daten, beispielsweise über die Materialzusammensetzung von Gebäuden, als weniger wichtig wahrgenommen $\mathrm{zu}$ werden. Weiters stellt die Abschätzung der Lebensdauern von Gebäuden in vielen Modellen eine Herausforderung dar. Durchschnittliche Lebensdauern für unterschiedliche Gebäudekategorien erscheinen für eine umfassende Beschreibung der Dynamiken im Gebäudebestand zu kurz gegriffen (Kohler und Yang 2007). Wie in Kleemann et al. (2015) beschrieben, ist die Qualität von statistischen Daten über Zusammensetzung von Abbruchabfällen oft mangelhaft. Hinsichtlich der Abbruchaktivität gibt es selbst in gut verwalteten Städten wie Wien oft nur rudimentäre Aufzeichnungen. Daher sind Alternativen $\mathrm{zu}$ statistischen Aufzeichnungen notwendig, die dabei helfen können, die Abbruchaktivität und die damit verbundenen Abfallmengen und -qualitäten abzuschätzen. Die vorliegende Arbeit präsentiert durch einen interdisziplinären Ansatz eine solche Alternative. Die Verwendung von Luftbildern, Geodaten und Materialdaten ermöglicht es, die notwendigen Informationen $\mathrm{zu}$ generieren und liefert damit die Grundlage, kontinuierlich Daten zu erheben, um zukünftig anfallende Abfallströme zu prognostizieren.

\section{Materialien und Methode}

In der vorliegenden Studie werden unterschiedliche Datenquellen verwendet, um Menge und Zusammensetzung von Abbruchabfällen aus Gebäuden in Wien zu bestimmen. Dabei kommen innovative Methoden aus zwei unterschiedlichen Disziplinen zum Einsatz. Die Abbruchaktivität ( $\mathrm{m}^{3}$ abgebrochener Bruttorauminhalt) unterschiedlicher Gebäudekategorien in Wien wird einerseits auf Basis von Aufzeichnungen über Abbruchanzeigen seitens der Stadt Wien und andererseits auf Basis von Bildmatching-basierter Veränderungsdetektion evaluiert. Alle Daten werden dabei in einem GIS (Geoin- 


\begin{tabular}{|c|c|c|c|c|}
\hline $\begin{array}{c}\text { Spezifische } \\
\text { Materialintensitäten } \\
{\left[\mathrm{kg} / \mathrm{m}^{3}\right] \text { unterschiedlicher }} \\
\text { Gebäudekategorien }\end{array}$ & كُ & $\begin{array}{l}\text { Volumen abgebrochenes } \\
\text { Gebäudevolumen }\left[\mathrm{m}^{3}\right] \text { je } \\
\text { Gebäudekategorie }\end{array}$ & & $\begin{array}{l}\text { Menge [kg] und Qualität } \\
\text { von Baurestmassen aus } \\
\text { Gebäude-abbrüchen }\end{array}$ \\
\hline
\end{tabular}

Abb. 1 Schematische Darstellung der Vorgangsweise

formationssystem) bearbeitet. $15 \mathrm{Ge}$ bäudekategorien, unterschieden durch Nutzung (Wohnen, Gewerbe, Industrie) und Bauperiode (bis 1918, 1919-1945, 1946-1976, 1977-1996, ab 1997) werden durch bestimmte spezifische Materialintensitäten $\left(\mathrm{kg} / \mathrm{m}^{3}\right.$ Bruttorauminhalt) charakterisiert. Durch die Kombination der spezifischen Materialintensitäten mit den abgebrochenen Gebäudevolumina wird auf Menge und Zusammensetzung von Abbruchabfällen geschlossen (Abb. 1).

\subsection{Aufzeichnungen über Abbruchanzeigen}

Gebäudeabbrüche in Wien müssen bei der Baubehörde angezeigt werden. Nur in besonderen Fällen, beispielsweise wenn sich das Gebäude in einer Schutzzone befindet, ist eine Genehmigung für den Abbruch notwendig. Die Magistratsabteilung 37 - Baupolizei (MA 37) führt Aufzeichnungen über die eingegangenen Abbruchanzeigen in Form einer Dokumentation der betroffenen Adressen. Eine Abbruchstatistik mit näheren Informationen über das abgebrochene Gebäude (Größe, Nutzung, Bauperiode) wird nicht geführt. Um die benötigten Informationen über die abgebrochenen Gebäude zu bekommen, werden daher die Adressdaten der Abbruchanzeigen mithilfe von Orthofotos überprüft. Anhand der Orthofotos können Abbrüche verifiziert werden und die Ausmaße des Abbruchs festgestellt werden. Geodaten unterschied- licher Wiener Magistratsabteilungen liefern die benötigten Informationen zu Größe, Nutzung und Bauperiode der abgebrochenen Gebäude. Abb. 2 zeigt einen großen Gebäudekomplex nach (links) und vor (Mitte) dem Abbruch sowie die Geodaten als Polygone der Gebäude (rechts).

Die so erhobenen Daten, mit Information über Größe, Nutzung und Bauperiode der abgebrochenen Gebäude, zifischen Materialintensitäten $\left(\mathrm{kg} / \mathrm{m}^{3}\right.$ Bruttorauminhalt BRI) der unterschiedlichen Gebäudekategorien (siehe Abschn. 2.3.) kombiniert, um Menge und Zusammensetzung der Abbruchabfälle abzuschätzen.

\subsection{Abgebrochenes Volumen \\ (Bildmatching-basierte \\ Veränderungsdetektion)}

Über einen Zeitraum von etwa zwei Jahren wurden Abbrüche im Stadtgebiet notiert, die von Institutsmitarbeitern zufällig wahrgenommen wurden. Dabei zeigte sich, dass die beobachteten Abbrüche teilweise nicht durch die in Abschn. 2.1. beschriebene Erhebung erfasst wurden. Eine alternative Methode zur Erhebung der Abbruchaktivität, basierend auf Bildmatching-basierter Veränderungsdetektion, wurde daher getestet.

Mit der Publikation des semi-globalmatching durch Heiko Hirschmüller (Hirschmüller 2005, 2008) hat Bildmatching in der Ableitung von fläwerden in weiterer Folge mit den spe- chendeckenden Höhenmodellen aus Luftbildern stark an Bedeutung gewonnen. Seither wird Bildmatching im wissenschaftlichen Bereich kontinuierlich weiterentwickelt, indem die verwendeten Algorithmen der jeweiligen Nutzung spezifisch angepasst und verändert werden. Einige Studien bedienen sich des Bildmatchingverfahrens als kostengünstigere Alternative zu Airborne Laserscanning, um Höhenmodelle zu erstellen (Brenner 2005). Gebäudemodelle durch das Bildmatchingverfahren abzuleiten ist ebenfalls eine bereits etablierte Vorgehensweise und wurde beispielsweise verwendet, um den Zerstörungsgrad nach Erdbeben zu beurteilen (Turker und Cetinkaya 2005), die Entwicklung von städtischen Gebieten nachzuvollziehen (Paparoditis et al. 1998), oder um historische Siedlungsstrukturen $\mathrm{zu}$ rekonstruieren (Nebiker et al. 2014). Dense image matching ist mittlerweile in vielen fotogrammetrischen Standard-Softwarepaketen enthalten und ermöglicht es, digitale Oberflächenmodelle aus orientierten Luftbildern zu erstellen (Haala 2013). Digitale Oberflächenmodelle (DOM) sind Höhenmodelle, welche die Erdoberfläche sowie natürliche und anthropogene Objekte auf der Erdoberfläche (Gebäude, Vegetation etc.) abbilden. Im Gegensatz dazu stellen digitale Geländemodelle (DGM) ausschließlich die Erdoberfläche dar. Die Grundfunktion des Bildmatching besteht darin, korrespondierende Punkte in sich überlappenden Bildern zu finden. Aufgrund der bekannten inneren Orientierung der Kamera und der äußeren Orientierung der Luftbilder ist es möglich, die dreidimensionale Position von korrespondierenden Punkten im Raum zu berechnen. Aus der daraus entstehenden 3D-

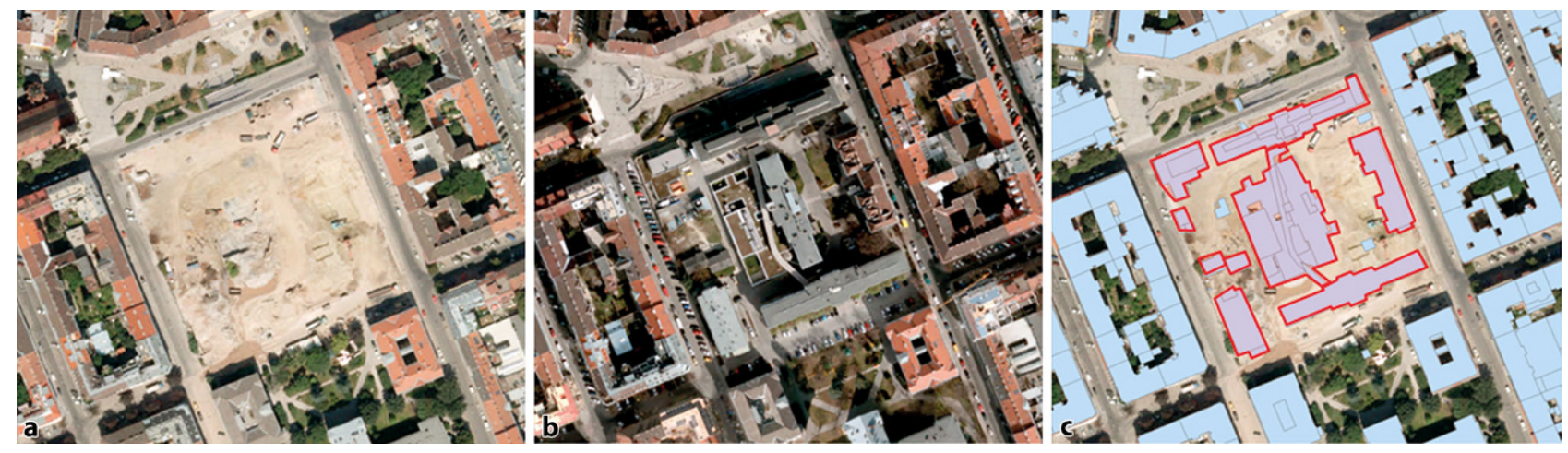

Abb. 2 Ermittlung des abgebrochenen Gebäudevolumens nach Gebäudekategorie basierend auf den Aufzeichnungen über Abbruchanzeigen in Wien, a Orthofoto (2013), b Orthofoto (2012) und c der digitalen Stadtkarte (2012) 


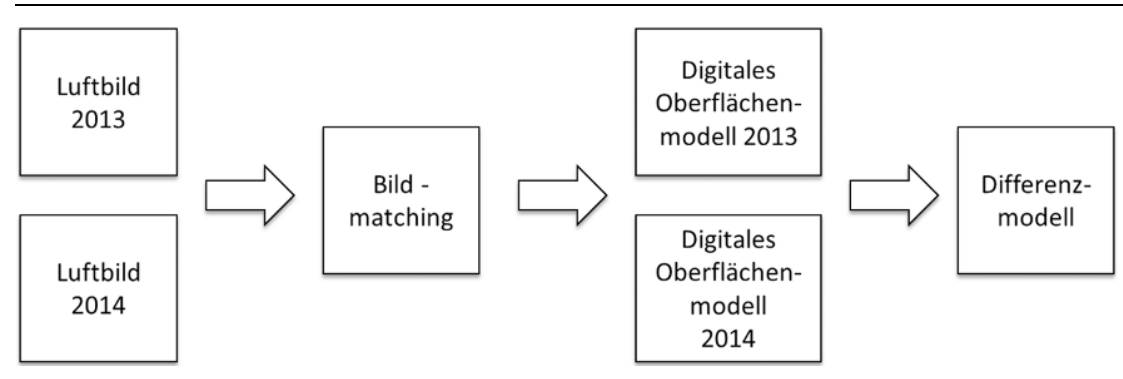

Abb. 3 Schematische Darstellung der Erstellung des Differenzmodells

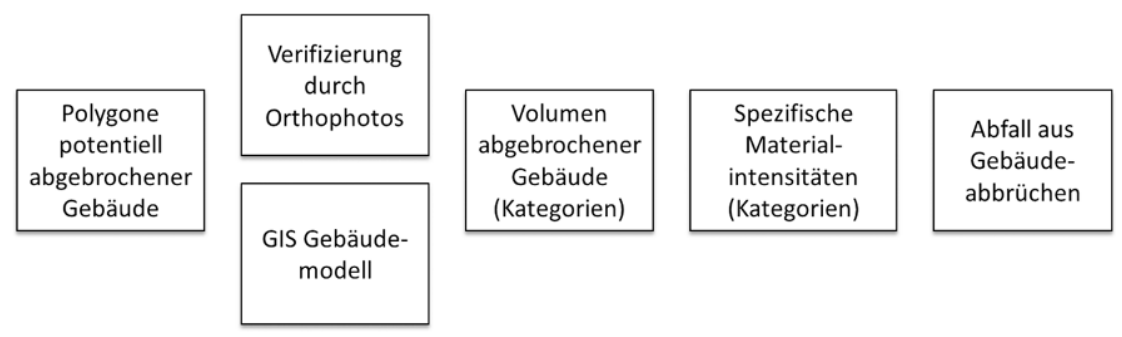

\section{Abb. 4 Schematische Darstellung der Vorgehensweise}

Punktwolke wird in der Folge ein regelmäßiges Höhenraster erstellt (in diesem Fall mit einer Auflösung von $25 \mathrm{~cm}$ ). Die Höhengenauigkeit im DOM beträgt für flache und gut texturierte Bereiche etwa $\pm 15 \mathrm{~cm}$. Unsicherheiten der Daten des Bildmatching gibt es einerseits in Häuserschluchten und Innenhöfen, da hier Bereiche oft nur in einem Bild sichtbar sind. Schattenbereiche weisen oft nur wenig Textur auf und können daher meist nicht genau berechnet werden. Gewässer können durch Wellengang, Spiegelungen und Reflexionen aus verschiedenen Blickwinkeln in unterschiedlichen Luftbildern eine andere Textur aufweisen und dadurch im DOM ebenfalls unsichere Höhenwerte liefern. Durch die Verwendung mehrerer stark überlappender Luftbilder können die nachteiligen Effekte reduziert werden. In dem bei der Magistratsabteilung 41 - Stadtvermessung Wien (MA 41) verwendeten Workflow wird die Verwendung stark überlappender Luftbilder zusätzlich zur DOM Erstellung auch dazu genutzt, einen Qualitätslayer zu berechnen (ebenfalls mit einer Auflösung von $25 \mathrm{~cm}$ ), mit dem es möglich ist, Bereiche mit sicheren und unsicheren Höheninformationen im DOM zu unterscheiden.

Eine Zielsetzung der MA 41 ist es, durch Vergleiche von Oberflächenmodellen mit dem 3D-Stadtmodell Veränderungen der Bebauung zwischen
Datenbestand und Naturstand festzustellen und diese Information für die Steuerung der Datenaktualisierung zu nutzen. Wird anstatt des 3D-Stadtmodells ein Bildmatching-DOM eines zweiten Bildflugs herangezogen, kann man für den Zeitraum zwischen den Bildflügen Hinweise auf die Veränderung der Bebauung ermitteln. Bei den Änderungen kann es sich um Neubauten, Aufstockungen oder Abbrüche handeln. Auf diese Weise können für einen definierten Zeitraum (Aufnahmezeitraum der Bildflüge) zuverlässige Hinweise auf stattgefundene Abbrüche generiert und in weiterer Folge das abgebrochene Gebäudevolumen festgestellt werden.

Für die vorliegende Studie wird das Bildmatching-DOM von 2013 von jenem von 2014 subtrahiert. In dem resultierenden Differenzmodell werden Bereiche, die 2013 höhere Höhenwerten als 2014 aufweisen, wie z. B. bei Gebäudeabbrüchen, durch negative Werte abgebildet (Abb. 3). Bereiche mit negativen Werten $a b-3 m$ wurden in einem nächsten Schritt in Polygone umgewandelt und anschließend Polygone ab einer Fläche von $30 \mathrm{~m}^{2}$ selektiert. Die Höhendifferenz von $3 \mathrm{~m}$ wurde festgelegt, um Abbrüche ab dieser Höhe (also ca. ab einem Geschoss) zu berücksichtigen. Die Fläche von $30 \mathrm{~m}^{2}$ wurde gewählt, um auch relativ kleine Gebäude (z.B. Gartenhäuser) berücksichtigen zu können. Andere Objekte wie Carports, Schuppen und Autos werden durch diese Maßnahmen von der weiteren Betrachtung ausgeschlossen. Der Normalized Difference Vegetation Index (NDVI) wurde dazu verwendet, um Vegetation von Bauwerken zu unterscheiden und in weiterer Folge ausschließen zu können. Wasserflächen und Glashäuser wurden durch Nutzung weiterer Geodaten ebenfalls ausgeschlossen.

In weiteren Schritten (Abb. 4) wird mithilfe von Orthofotos verifiziert, ob es sich bei der Höhenänderung tatsächlich um einen Gebäudeabbruch handelt und in welchem Ausmaß dieser stattgefunden hat. Durch die Verwendung vorhandener Geodaten kann auf Volumen und Kategorie (Bauperiode/Nutzung) des abgebrochenen Gebäudes am jeweiligen Standort rückgeschlossen werden. Kombiniert mit spezifischen Materialintensitäten der unterschiedlichen Gebäudekategorien kann auf Menge und Qualität von Abbruchabfällen geschlossen werden.

Abb. 5 zeigt die Ermittlung des abgebrochenen Gebäudevolumens nach Gebäudekategorie mithilfe der Polygone potenziell abgebrochener Gebäude in Orthofotos.

\subsection{Spezifische Materialintensitäten}

Neben Informationen über Anzahl und Größe der abgebrochenen Gebäude bedarf es zur Abschätzung von Menge und Zusammensetzung von Abbruchabfällen auch Informationen über die materielle Zusammensetzung der abgebrochenen Gebäude. Im Folgenden wird beschrieben, wie spezifische Materialintensitäten (ausgedrückt in $\mathrm{kg} / \mathrm{m}^{3}$ Bruttorauminhalt) für unterschiedliche Gebäudekategorien in Wien, die nach Bauperiode und Nutzung eingeteilt wurden, generiert wurden.

\subsubsection{Fallstudien}

14 Gebäude unterschiedlicher Nutzung und Bauperiode wurden hinsichtlich ihrer Materialzusammensetzung vor deren Abbruch untersucht. Einerseits wurden dabei vorhandene Unterlagen (Bestandspläne, Gutachten etc.) analysiert, andererseits wurden Begehungen und selektive Beprobungen im Gebäude durchgeführt. Bei Begehung und selektiver Beprobung wurden für unterschiedliche Gebäudebereiche bzw. Bauteile (Fußböden, Zwischenwände, Deckenabhängungen, Fenster, Türen, 


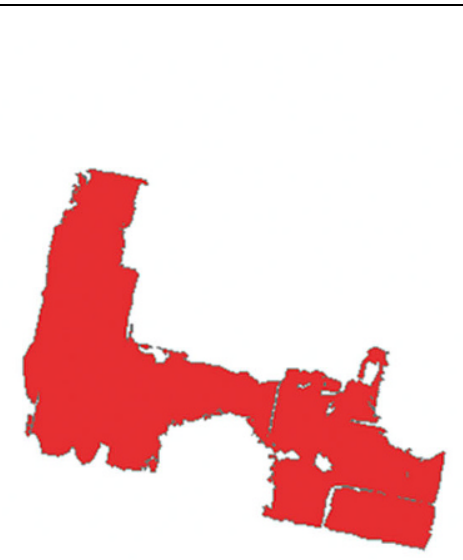

a
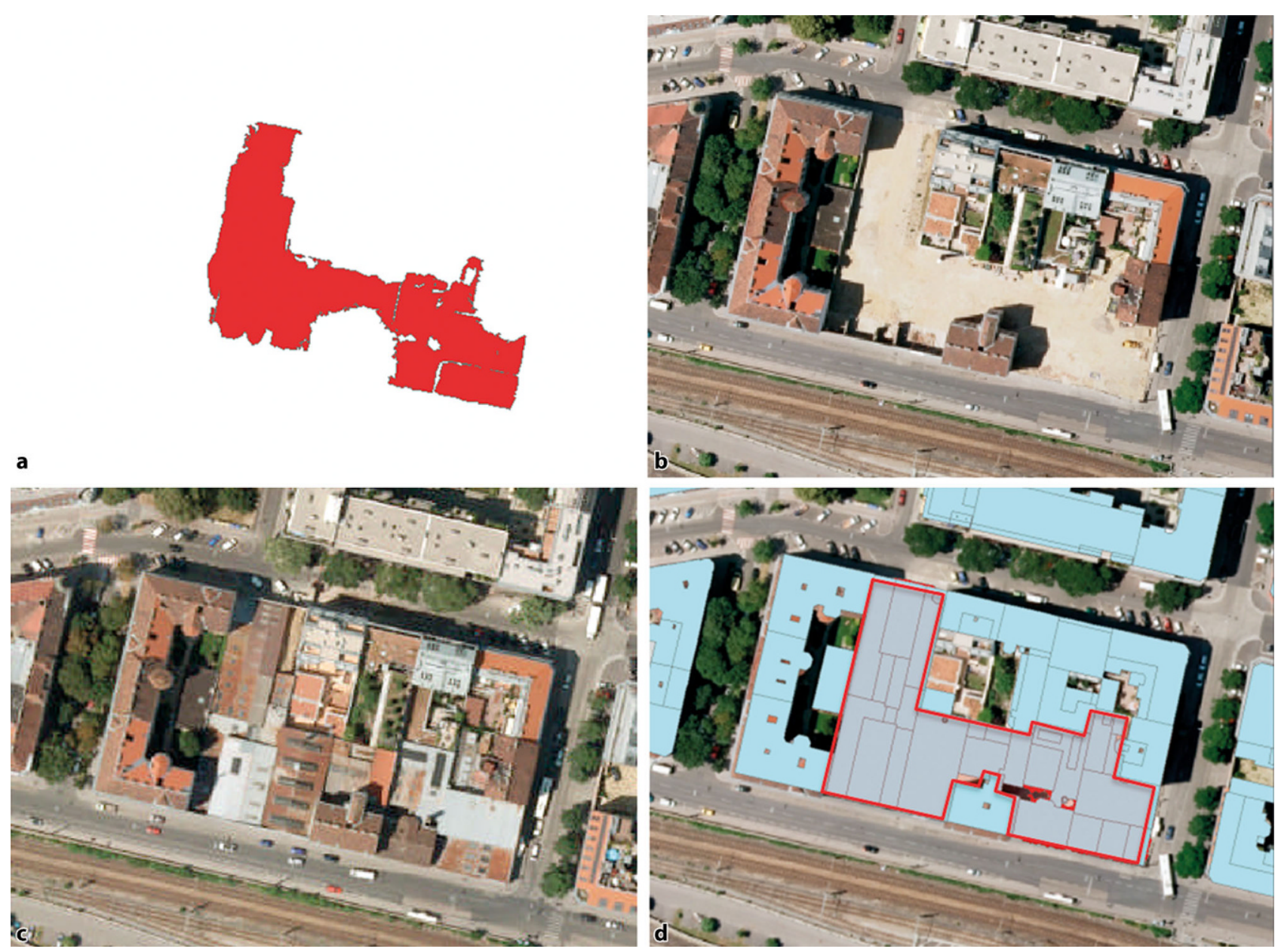

Abb. 5 Ermittlung des abgebrochenen Gebäudevolumens nach Gebäudekategorie mithilfe von a Polygonen potenziell abgebrochener Gebäude, b, c Orthofotos und d Geodaten (Gebäudeflächen der digitalen Stadtkarte)

Türzargen etc.), durch Demontage, Wiegung und/oder Vermessung, Daten zu deren Aufbau und materieller Zusammensetzung erhoben. Auf Basis der Volumina und der jeweiligen Materialdichte bzw. des jeweiligen Gewichts wurden Massen berechnet, um eine möglichst detaillierte Materialbilanz des Gebäudes zu erstellen (Kleemann et al. 2015).

\subsubsection{Daten aus Bauakten von Abbruchgebäuden}

In einem weiteren Schritt wurden Bauakten von bereits abgebrochenen Gebäuden, welche bei der Baupolizei (MA 37) angezeigt wurden, gesammelt und hinsichtlich ihrer Materialzusammensetzung analysiert. Abhängig von der Qualität der Bauakten konnten dabei Wand- und Deckenaufbauten sowie teilweise Dach- und Fußbodenkonstruktionen und die Art der Fenster bestimmt werden. Daten zur Material- zusammensetzung der Gebäude, welche nicht aus den Bauakten ersichtlich sind (z. B. Kunststoffe und Metalle in Installationen, Holz, Kunststoffe und Aluminium in Fenstern, Türen oder Fußbodenbelägen), wurden durch Informationen aus den Fallstudien ergänzt. Von rund 40 Gebäuden konnten die Bauakten herangezogen werden. Etwa 100 Bauakten gaben nicht ausreichend Informationen für eine befriedigende Analyse.

\subsubsection{Daten über Neubauten und Literaturrecherche}

Materialdaten aus Lebenszyklusanalysen des Österreichischen Instituts für Baubiologie und Bauökologie (IBO) und Informationen von Bauherren einzelner Neubauprojekte wurden analysiert und verwendet. Weiters wurden durch Literaturrecherche relevante Daten zur Materialzusammensetzung von unter- schiedlichen Gebäuden gesammelt und je nach Gültigkeit verwendet.

Tab. 1 zeigt die spezifischen Materialintensitäten der unterschiedlichen Gebäudekategorien, welche in der gegenständlichen Studie verwendet wurden. Diese basieren auf Kleemann et al. (2016a), wobei diese eigens für Wien erhoben wurden und nach Information der Autoren die beste verfügbare Information zur Materialzusammensetzung der unterschiedlichen Gebäudekategorien darstellen. Trotzdem ergeben sich Unsicherheiten sowohl durch die Kategorisierung als auch bei der Datenerhebung. Baupläne können sich beispielsweise von der tatsächlichen Situation vor Ort unterscheiden und bei Messungen vor Ort können Fehler passieren. Zusätzlich mussten in manchen Situationen Abschätzungen getroffen werden, da nicht jedes Bauteil vor Ort verwogen werden kann. Diese Unsicherheiten, welche in Kap. 4. weiter diskutiert werden, sollten bei 


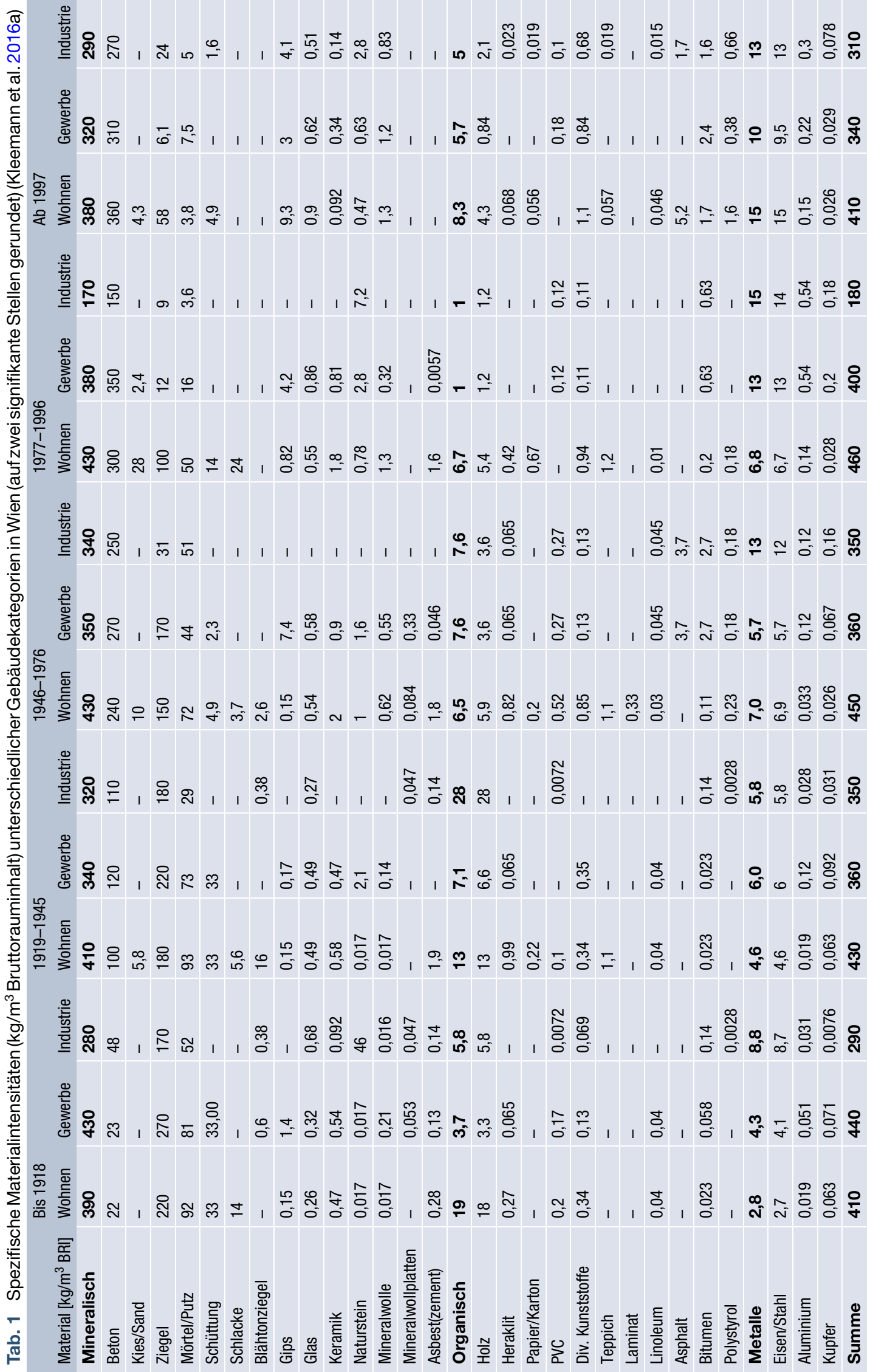




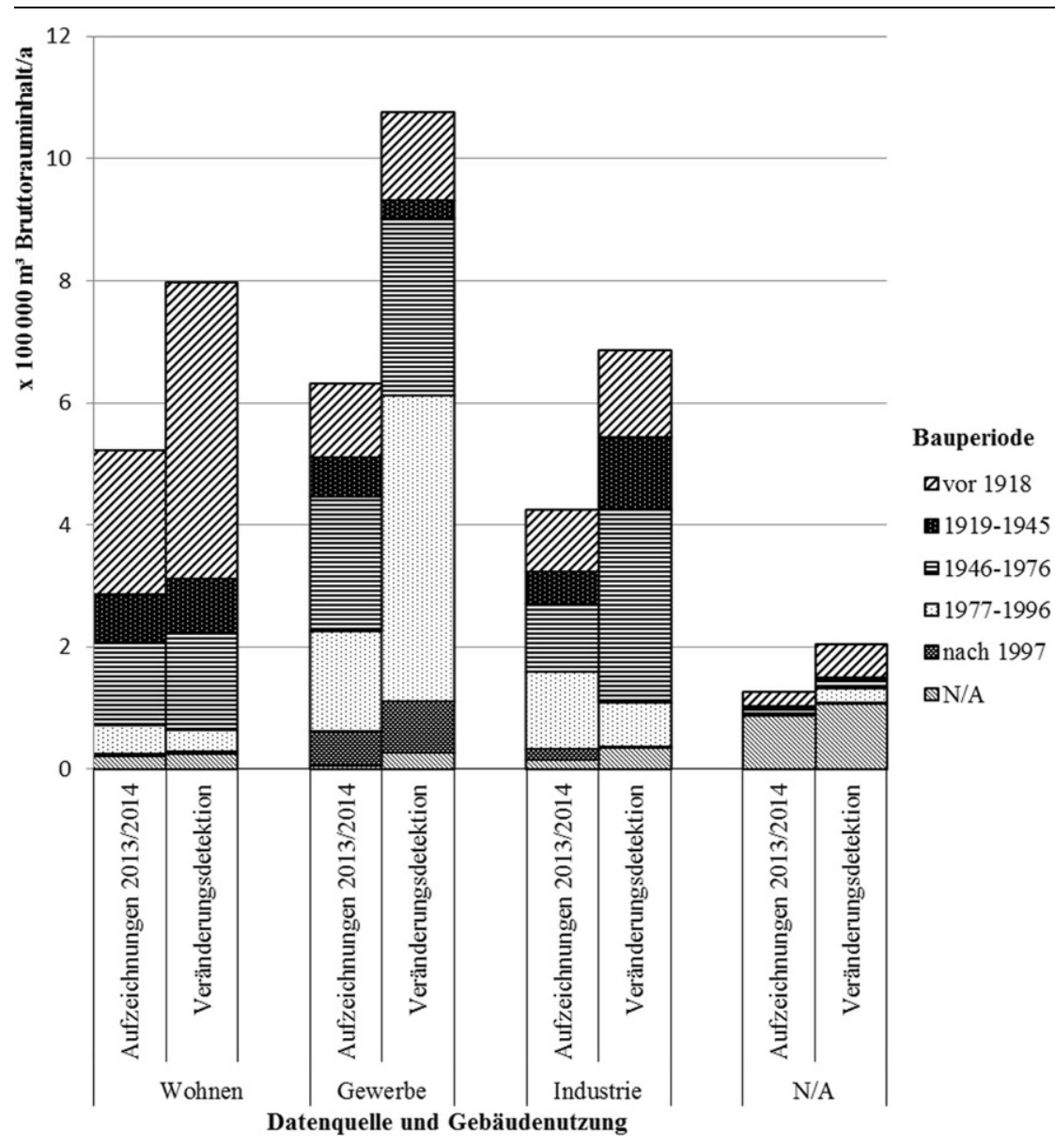

Abb. 6 Vergleich des abgebrochenen Volumens je Gebäudekategorie auf Basis von Aufzeichnungen über Abbruchanzeigen und Bildmatching-basierter Veränderungsdetektion

der Auswertung und den erhaltenen Ergebnissen jedenfalls bedacht werden.

\section{Resultate und Diskussion}

Basierend auf den Aufzeichnungen über Abbruchanzeigen der MA 37 der Jahre 2013 und 2014, Orthofotos von 2012 bis 2014, den Daten aus der Bildmatchingbasierten Veränderungsdetektion sowie Geodaten konnte das abgebrochene Gebäudevolumen unterschiedlicher Gebäudekategorien berechnet werden. In Kombination mit spezifischen Materialintensitäten der unterschiedlichen Gebäudekategorien konnte in weiterer Folge auf Menge und Qualität von Abfällen aus dem Gebäudeabbruch in Wien geschlossen werden.

\subsection{Abgebrochenes Gebäudevolumen}

Mittels Bildmatching-basierter Veränderungsdetektion wurde ein abgebrochenes Gebäudevolumen von 2,8 Mio. raum zwischen den Bildflügen (Juli 2013 und Juni 2014) nur 10,5 Monate betrug.

Für einen kleinen Teil des Volumens sind weder Informationen zur Nutzung noch zur Bauperiode vorhanden. Für diesen Teil wurde angenommen, dass die Verteilung der Gebäudekategorien gleich dem des gesamten abgebrochenen Volumens ist.

Der in Abb. 7 angestellte Vergleich der beiden Datenquellen unterstreicht einerseits, dass durch die Veränderungsdetektion eine deutlich höhere Gesamtzahl an Abbrüchen berücksichtigt wird. Andererseits zeigt das Ergebnis auch den großen Einfluss weniger, großer Abbruchvorhaben auf das Gesamtvolumen abgebrochener Gebäude. 6 bis $7 \%$ der Abbrüche (rund $30 \mathrm{Ob}$ jekte) repräsentieren etwa $50 \%$ des abgebrochenen Gesamtvolumens.

\subsection{Menge und Qualität von Abfällen aus dem Gebäudeabbruch}

Durch die Kombination des abgebrochenen Gebäudevolumens der unterschiedlichen Gebäudekategorien mit den jeweiligen spezifischen Materialintensitäten können Menge und Qualität von Abfällen abgeschätzt werden. Da die Daten der Bildmatching-basierten Veränderungsdetektion umfassendere Ergebnisse als jene der Aufzeichnungen über Abbruchanzeigen lieferten, wurden letztere in weiterer Folge nicht mehr verwendet.

Gemäß den angestellten Berechnungen entstehen in Wien pro Jahr rund 1,1 Mio. t Abbruchabfälle aus dem Gebäudebereich, was etwa $600 \mathrm{~kg}$ pro Person und Jahr entspricht. Vergleicht man diese Zahlen mit dem Gesamtlager an Materialien in Wiens Gebäuden (210t pro Person nach Kleemann et al. 2016a), ergibt sich eine Abbruchrate von etwa $0,3 \%$. Eine grobe Berechnung, auf Basis von Daten aus dem Gebäude- und Wohnungsregisters der MA 37, ergab im Vergleich dazu eine Neubaurate von $0,8 \%$ und einen Bedarf an Baumaterialien von insgesamt etwa 2,9 Mio. $\mathrm{t}$ jährlich bzw. $1600 \mathrm{~kg}$ pro Person und Jahr.

Tab. 2 zeigt die errechneten ProKopf-Mengen unterschiedlicher Materialien. Der Großteil der Materialien ist mineralisch ( $>95 \%)$, wobei Beton (45\%), Ziegel (30\%) sowie Mörtel und Putz (13\%) die Hauptfraktionen darstellen. Nur ein sehr geringer Anteil $(<5 \%)$ der Gesamtmenge der Abbruch- 


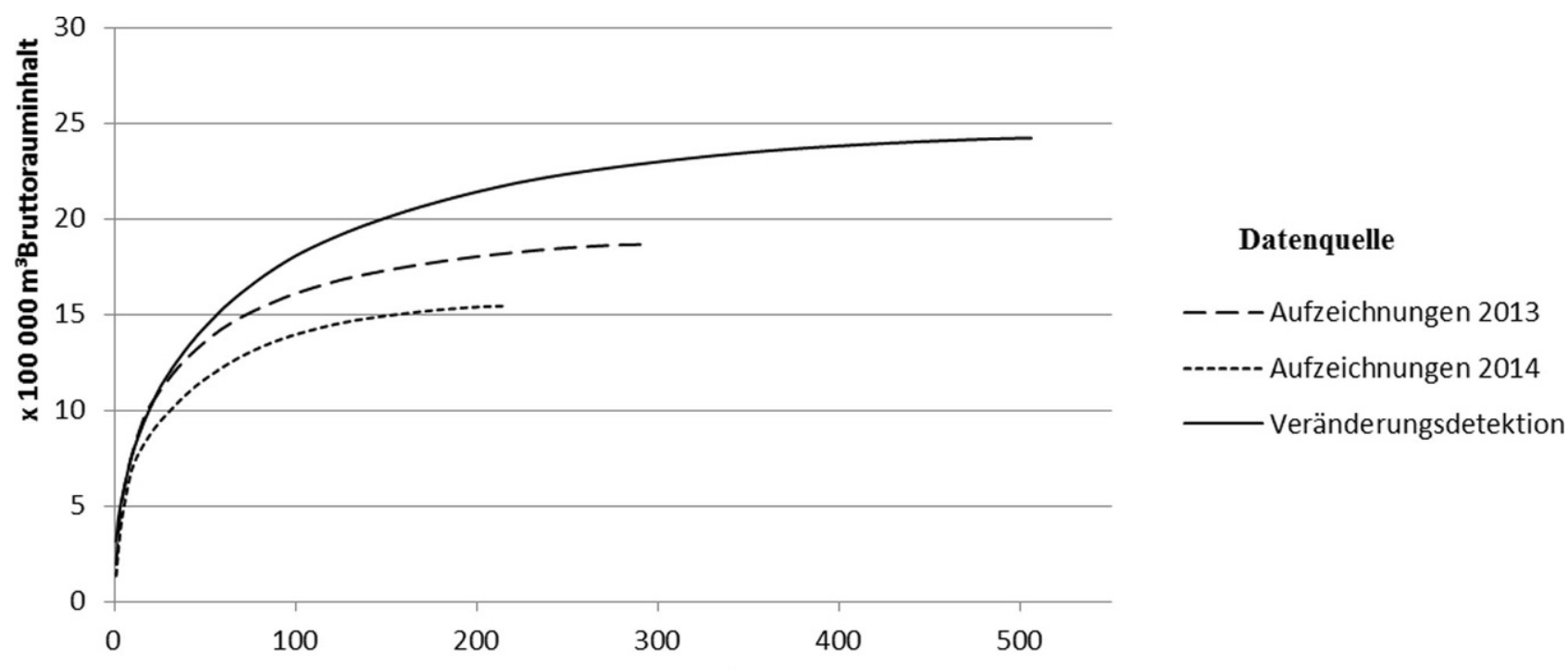

Anzahl der Abbruchprojekte

Abb. 7 Vergleich derErgebnisse derBildmatching-basierten Veränderungsdetektion mitErgebnissen derAbbruchstatistiken(Kleemann et al. 2016b)

Tab. 2 Abfallmengen ausgewählter Materialien aus dem Gebäudeabbruch [kg/Einwohner/Jahr]

\begin{tabular}{|c|c|c|c|c|c|}
\hline \multicolumn{6}{|l|}{ kg/Einwohner/Jahr } \\
\hline Mineralisch & 570 & Organisch & 15 & Metalle & 12 \\
\hline Beton & 280 & Holz & 11 & Eisen/Stahl & 12 \\
\hline Ziegel & 180 & Asphalt & 1,4 & Aluminium & 0,26 \\
\hline Mörtel/Putz & 76 & Bitumen & 1,3 & Kupfer & 0,15 \\
\hline Schüttung & 15 & Div. Kunststoffe & 0,35 & - & - \\
\hline Naturstein & 6 & PVC & 0,27 & - & - \\
\hline Schlacke & 5 & Heraklit & 0,25 & - & - \\
\hline Gips & 2,8 & Teppich & 0,19 & - & - \\
\hline Kies/Sand & 2,6 & Polystyrol & 0,1 & - & - \\
\hline Blähtonziegel & 1,2 & Papier/Karton & 0,045 & - & - \\
\hline Keramik & 0,86 & Linoleum & 0,037 & - & - \\
\hline Glas & 0,65 & Laminat & 0,032 & - & - \\
\hline Asbest(zement) & 0,43 & - & - & - & - \\
\hline Mineralwolle & 0,33 & - & - & - & - \\
\hline Mineralwollplatten & 0,078 & - & - & Summe & 600 \\
\hline
\end{tabular}

materialien besteht aus organischen Materialien oder Metallen, wobei Holz, Eisen/Stahl jeweils dominieren.

Der Großteil der Abbruchabfälle wird zur Aufbereitung außerhalb der Stadt transportiert. Aufbereiteter Beton findet üblicherweise im Straßenbau Anwendung, während Ziegel in Wien hauptsächlich in der Zementindustrie als Tonersatz verwendet werden. Kleinere Mengen werden zu Pflanzensubstrat oder Tennissand aufbereitet. Die Verwendung des Ziegelmaterials in der Zementindustrie ist ein Spezifikum für den Wiener Raum. Organisches Material wird meist thermisch verwertet. Holz wird in der Plattenindustrie teilweise auch materiell recycelt. Aufgrund ihres
Wertes werden Metalle nahezu zu $100 \%$ recycelt.

\subsection{Unsicherheiten}

Sowohl die Resultate über die Abbruchaktivität als auch jene über die Materialintensitäten unterliegen Unsicherheiten. Für die Abschätzung der Gesamtmenge an Abfällen wird eine Unsicherheit von unter $15 \%$ angenommen. Für einzelne Materialien, speziell solche in geringer Konzentration (z.B. Aluminium), müssen höhere Unsicherheiten in Kauf genommen werden. Was die angewandte Methode auszeichnet, ist die Aktualität der verwendeten $\mathrm{Da}$ ten.

\section{Schlussfolgerungen}

Die präsentierte Methode zeigt, wie durch einen interdisziplinären Ansatz Abfälle bzw. Sekundärressourcen, die durch Abbrüche in Wien zur Verfügung stehen, abgeschätzt werden können. Der beschriebene Ansatz zeigt, dass Werkzeuge der Fernerkundung dabei helfen können, die Abbruchaktivität in Wien nachzuvollziehen. In Kombination mit spezifischen Materialintensitäten unterschiedlicher Gebäudekategorien ist es weiters möglich, auf Menge und Qualität von Abfällen aus dem Gebäudeabbruch zu schließen. Die Methode ist praktisch anwendbar und kann aufgrund der herrschenden 
Rahmenbedingungen relativ einfach in bestehende Arbeitsabläufe integriert und so implementiert werden. Dadurch würde ein kontinuierliches Monitoring des Gebäudebestands ermöglicht und es könnten regionale Vergleichszahlen $\mathrm{zu}$ Abfallstatistiken generiert werden. Abfallrelevante Daten werden in Österreich zentral über das Elektronische Datenmanagement (EDM) erfasst und ausgewertet, Aussagen über bestimmte Regionen, beispielsweise Ballungsgebiete, können aber nur eingeschränkt getroffen werden. Für den Anfall von Abbruchabfällen kann die vorgestell- te Methode daher regionalspezifische Informationen liefern. Kontinuierlich angewandt ermöglicht die Methode das Monitoring der Entwicklung des Gebäudebestands und in weiterer Folge eine Prognose über Dynamiken der Stadtentwicklung. Weiters können effektive Maßnahmen zur Ausrichtung der Abfallwirtschaft und des Ressourcenmanagements im Sinne einer Kreislaufwirtschaft getroffen werden.

Funding Open access funding provided by TU Wien (TUW).
Open Access Dieser Artikel wird unter der Creative Commons Namensnennung 4.0 International Lizenz (http:// creativecommons.org/licenses/by/4. $0 /$ deed.de) veröffentlicht, welche die Nutzung, Vervielfältigung, Bearbeitung, Verbreitung und Wiedergabe in jeglichem Medium und Format erlaubt, sofern Sie den/die ursprünglichen Autor(en) und die Quelle ordnungsgemäß nennen, einen Link zur Creative Commons Lizenz beifügen und angeben, ob Änderungen vorgenommen wurden.
Bergsdal, H., Bohne, R. A., \& Brattebø, H. (2007): Projection of Construction and Demolition Waste in Norway. Journal of Industrial Ecology, 11(3), 27-39. https://doi.org/10.1162/ jiec.2007.1149

BMLFUW (2011): Bundes-Abfallwirtschaftsplan 2011. Vienna: Bundesministerium für Land- und Forstwirtschaft, Umwelt und Wasserwirtschaft (BMFLUW).

BMLFUW (2015): Bundes-Abfallwirtschaftsplan 2015. Vienna: Bundesministerium für Land- und Forstwirtschaft, Umwelt und Wasserwirtschaft (BMFLUW).

Brenner, C. (2005): Building reconstruction from images and laser scanning. International Journal of Applied Earth Observation and Geoinformation, 6(3), 187-198.

Cheng, J. C., \& Ma, L. Y. (2013): A BIM-based system for demolition and renovation waste estimation and planning. Waste Management, 33(6), 1539-1551.

Cochran, K. M., \& Townsend, T. G. (2010): Estimating construction and demolition debris generation using a materials flow analysis approach. Waste Management, 30(11), 2247-2254. https://doi.org/10.1016/j.wasman.2010.04.008 Directive 2008/98/EC of the European Parliament and of the Council of 19 November 2008 on waste and repealing certain Directives (2008) European Comission (2014): Construction and Demolition Waste (CDW). Retrieved 05.03., 2014, from http://ec.europa.eu/environment/waste/ construction_demolition.htm

Fatta, D., Papadopoulos, A., Avramikos, E. Sgourou, E., Moustakas, K., Kourmoussis, F., ... Loizidou, M. (2003): Generation and management of construction and demolition waste in Greece - an existing challenge. Resources, conservation and recycling, 40(1), 81-91. https://doi. org/10.1016/S0921-3449(03)00035-1
Haala, N. (2013): The landscape of dense image matching algorithms. Photogrammetric Week, 13, 271-284.

Hashimoto, S., Tanikawa, H., \& Moriguchi, Y. (2009): Framework for estimating potential wastes and secondary resources accumulated within an economy - A case study of construction minerals in Japan. Waste Management, 29(11), 2859-2866.

Hirschmüller, H. (2005): Accurate and efficient stereo processing by semi-global matching and mutual information. Paper presented at the Computer Vision and Pattern Recognition, San Diego, CA, USA.

Hirschmüller, H. (2008): Stereo processing by semi-Global matching and mutual information. IEEE Transactions on Pattern Analysis and Machine Intelligence, 30(2), 328-341.

Hu, D., You, F., Zhao, Y., Yuan, Y., Liu, T., Cao A., ... Zhang, J. (2010): Input, stocks and output flows of urban residential building system in Beijing city, China from 1949 to 2008. Resources, conservation and recycling, 54(12), 1177-1188. https://doi.org/10.1016/j.resconrec.2010.03.011 Kleemann, F., Aschenbrenner, P., \& Lederer, J. (2015): Methode zur Bestimmung der Materialzusammensetzung von Gebäuden vor dem Abbruch. Österreichische Wasser- und Abfallwirtschaft, 21-27. https://doi.org/10.1007/s00506014-0203- $\mathrm{x}$

Kleemann, F., Lederer, J., Rechberger, H., \& Fellner, J. (2016): GIS-based Analysis of Vienna's Material Stock in Buildings. Journal of Industrial Ecology, n/a-n/a. https://doi.org/10.1111/jiec. 12446

Kleemann, F., Lehner, H., Szczypińska, A., Lederer, J., \& Fellner, J. (2016): Using change detection data to assess amount and composition of demolition waste from buildings in Vienna. Re- sources, conservation and recycling. https://doi. org/10.1016/j.resconrec.2016.06.010

Kohler, N., \& Yang, W. (2007): Long-term management of building stocks. Building Research \& Information, 35(4), 351-362.

De Melo, A. B., Goncalves, A. F., \& Martins, I. M. (2011): Construction and demolition waste generation and management in Lisbon (Portugal). Resources, conservation and recycling, 55(12), 1252-1264

Nebiker, S., Lack, N., \& Deuber, M. (2014): Building change detection from historical aerial photographs using dense image matching and object-based image analysis. Remote Sensing, 6(9), 8310-8336.

Paparoditis, N., Cord, M., Jordan, M., \& Cocquerez, J.-P. (1998): Building detection and reconstruction from mid-and high-resolution aerial imagery. Computer vision and image understanding, 72(2), 122-142.

Solís-Guzmán, J., Marrero, M., Montes-Delgado, M. V., \& Ramírez-de-Arellano, A. (2009): A Spanish model for quantification and management of construction waste. Waste Management 29(9), 2542-2548.

Tanikawa, H., \& Hashimoto, S. (2009): Urban stock over time: spatial material stock analysis using 4d-GIS. Building Research \& Information, 37(5-6), 483-502.

Turker, M., \& Cetinkaya, B. (2005): Automatic detection of earthquake-damaged buildings using DEMs created from pre-and post-earthquake stereo aerial photographs. International Journal of Remote Sensing, 26(4), 823-832.

Yuan, H., \& Shen, L. (2011): Trend of the research on construction and demolition waste management. Waste Management, 31(4), 670-679. 